УДК 676.2.056-55

\title{
ПРИМЕНЕНИЕ СОВРЕМЕННЫХ МЕТОДОВ ЦИФРОВОЙ ОБРАБОТКИ СИГНАЛОВ В АСУТП БУМАЖНОГО ПРОИЗВОДСТВА
}

\author{
Лысова Наталья Владимировна \\ аспирант \\ Научный руководитель: Мясникова Нина Владимировна \\ д.т.н., профессор \\ ФГБОУ ВО «Пензенский государственный университет»
}

Аннотация: Современный этап развития АСУ ТП характеризуется применением сложных методов обработки информации. Работа посвящена исследованию методов экстремальной фильтрации (ЭФ) и декомпозиции на эмпирические моды (EMD) для формирования диагностических признаков, используемых в бумажном производстве. Приведены информативные составляющие, для построения прогноза, который может использоваться для изменения регулятора в системе.

Ключевые слова: Декомпозиция на эмпирические моды (EMD), экспресс-анализ, экстремальная фильтрация (ЭФ), Автоматизированная система управления технологическим процессом (АСУ ТП), бумагоделательная машина (БДМ).

\section{APPLICATION OF MODERN METHODS OF DIGITAL SIGNAL PROCESSING IN AUTOMATED CONTROL SYSTEMS OF PAPER PRODUCTION}

\author{
Lysova Natalia Vladimirovna \\ Scientific adviser: Myasnikova Nina Vladimirovna
}

\begin{abstract}
The current stage of development of automated process control systems is characterized by the use of complex methods of information processing. The work is devoted to the study of methods of extreme filtration and decomposition into empirical modes (EMD) for the formation of diagnostic features used in paper production. The informative components for constructing a forecast that can be used to change the regulator in the system are given.
\end{abstract}

Key words: Decomposition into empirical modes, express analysis, extreme filtration, Automated Process Control System, Paper Machine. 
Современный этап развития автоматизированных систем управления (АСУ) характеризуется применением сложных методов обработки информации, таких как: выделение трендовой составляющей; выделение информативной составляющей; сжатие и восстановление для хранения трендов и передачи по каналам связи; анализ, обработка и принятие решений в режиме реального времени; прогнозирование для своевременного принятия решений и предотвращения аварийных ситуаций или для адаптивного управления при изменении параметров объекта.

В настоящее время уже есть опыт применения сложных алгоритмов, например, для выделения трендовой и периодических составляющих применялся метод сингулярного разложения с последующим использованием тренда для прогноза поведения системы $[1,2]$. Однако трудоемкость этого метода не позволяет использовать результаты обработки в управлении объекта, они являются инструментом анализа регулирования процессов и качества работы системы.

Менее трудоемкими методами выделения таких составляющих являются декомпозиция на эмпирические моды и экстремальная фильтрация [3,4]. Применение этих методов в системах позволит существенно сократить время обработки, а экстремальная фильтрация допускает адаптацию к режиму реального времени.

Метод декомпозиции на эмпирические моды (EMD) является в настоящее время одним из самых востребованных в области цифровой обработки сигналов. Этот подход обладает высокой степенью адаптации к исследуемым сигналам, что позволяет проводить точное оценивание реальных свойств процессов, в том числе и нестационарных, улавливать незначительные изменения. Ключевым моментом является использование «декомпозиции на эмпирические моды», с помощью которой любой сложный сигнал может быть разложен на конечное и часто довольно малое число «эмпирических мод», каждая из которых содержит определенную информацию об исследуемом процессе.

Развитие систем автоматизированного управления технологическими процессами (АСУ ТП) вплотную связано с совершенствованием компьютерных технологий. В последнее десятилетие отмечается интенсивный процесс внедрения SCADA-систем. Система SCADA - процесс сбора информации реального времени с удаленных точек (объектов) для обработки, анализа и управления удаленными объектами. Он обусловлен возможностями современной измерительной базы, позволяющей не только выполнять 
измерения в полном объеме и с высокой точностью, но и передавать их в компьютер с помощью разнообразных телекоммуникационных средств. Информационной базой для работы SCADA-систем являются результаты измерений контрольно-измерительного оборудования, установленного на технологических объектах. Современная АСУ ТП представляет собой многоуровневую человеко-машинную систему управления. В функциональной структуре АСУ ТП с точки зрения пользователей можно выделить основные функциональные подсистемы, такие как сбора и первичной обработки информации, архивирования, автоматического регулирования и т.д. Современные SCADA-системы обеспечивают сбор и долгосрочное хранение трендов всех технологических параметров для последующего анализа в случае необходимости. Тренд представляет собой массив точек переменных, каждая из которых записывается в реальной системе в память компьютера через определенные интервалы времени. Анализ трендов необходим для повышения надежности работы объекта, увеличения его КПД и т.д.

Хотя данные такого типа хорошо сжимаются архиваторами, имеется возможность сократить объем исходных трендов, а, следовательно, и сжатых данных за счет предварительной обработки.

Для этого предлагается:

- исходный тренд, за определенный временной промежуток подвергнуть низкочастотной фильтрации;

- из отфильтрованного сигнала выделить экстремумы;

- сохранить в преобразованном тренде только экстремальные значения переменных $t_{i э}, x_{i э}$;

- впоследствии разархивировать тренд и восстановить по предлагаемой далее методике.

Рассмотрим реализацию данного алгоритма на примере производства бумаги. На рис. 1 представлена схема управления плотностью бумажной массы.

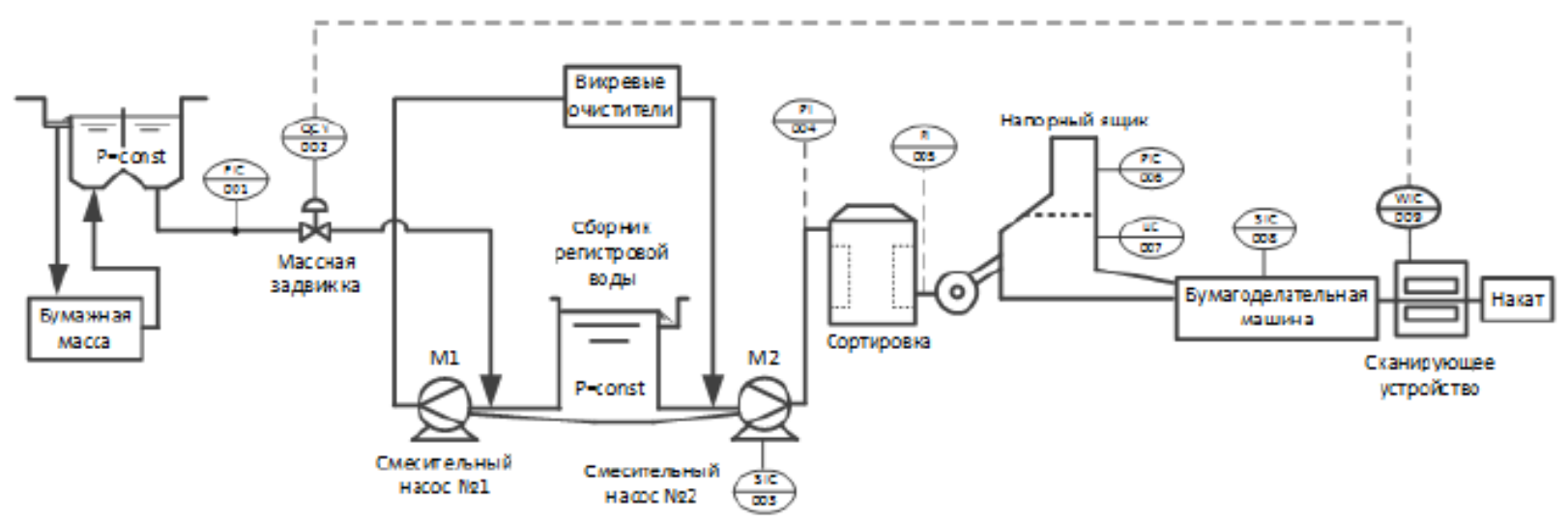

Рис. 1. Схема управления плотностью $1 \mathrm{м}^{2}$ бумажного полотна 
Покажем возможность применения предложенной методики. На рис. 2 приведены тренды, регистрируемые сканером. При накате массы наблюдается переходной процесс измерительного тракта, поэтому, всплески на графике не отражают истинной массы полотна [5].

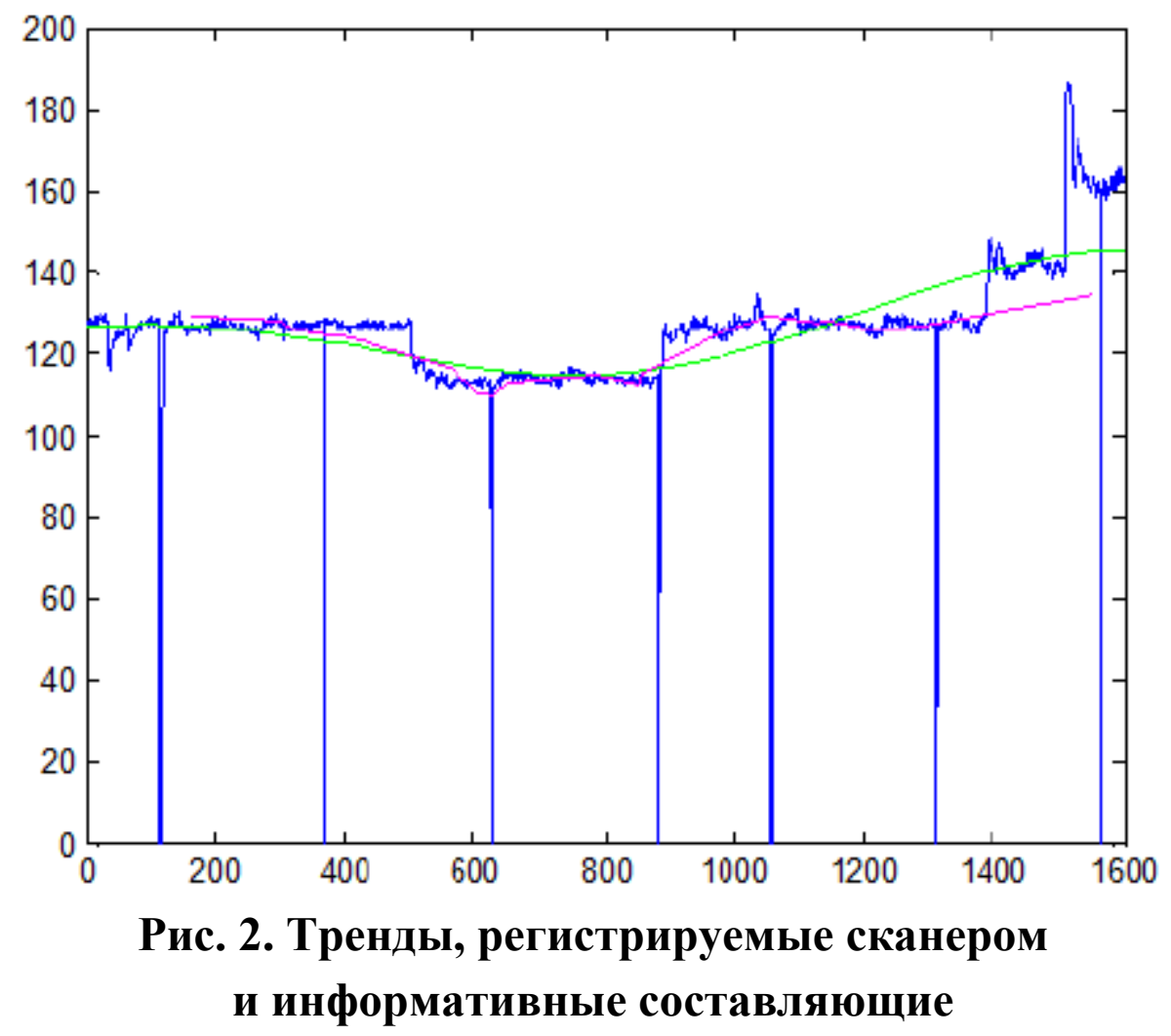

Показаны информативные составляющие, выделенные обоими методами. По выделенным данным на предыдущем участке может быть построен прогноз, который может использоваться для изменения уставок массовой задвижки, что повысит качество продукции.

Применение перечисленных алгоритмов позволяет использовать методы экстремальной фильтрации в системах реального времени, что дает возможность обеспечить своевременное обнаружение изменений всех быстропеременных процессов. Это способствует своевременному принятию решений об изменении уставки, что позволит улучшить качество производимой бумаги.

\section{Список литературы}

1. Лысова Н.В., Семенов А.Д., Волков В.В., Каргин В.М. Повышение точности выделения трендовой составляющей временного ряда $\mathrm{c}$ использованием сингулярного разложения // Проблемы автоматизации и 
управления в технических системах: сб.ст. Международной научнотехнической конференции (г.Пенза, 23-25 апреля 2013г.) под ред. д.т.н., проф. М.А. Щербакова. - Пенза: Изд-во ПГУ, 2013. - С. 174-176.

2. Лысова Н.В., Никулин С.В. Помехоустойчивое экстремальное регулирование инерционным объектом с запаздыванием // Современные технологии, в задачах управления, автоматики и обработки информации: Сборник трудов XXIII Международного научного технического семинара, 1420 сентября 2014г., Алушта. - М.: ИКД «Зеркало-М», 2014. - С. 19.

3. Экспресс-анализ сигналов в инженерных задачах: монография / Мясникова Н.В., Берестень М.П., Ципин Б.В., Мясникова М.Г. -Москва: ФИЗМАТЛИТ, 2016. 184 с.

4. Чеков П.Г. Технологии цифровой обработки сигналов в управлении бесплатформенными инерциальными системами // Информационные технологии в проектировании и производстве. - 2003. - №2. - С. 63-66.

5. Лысова Н.В., Мясникова Н.В. Регулирование веса бумажного полотна на основе метода экстремальной фильтрации для систем реального времени с помощью покадровой обработки сигнала // Инженерный вестник Дона. - 2019. №8. URL: ivdon.ru/ru/magazine/archive/N5y2019/5882.

(С Н.В. Лысова, Н.В. Мясникова, 2021 